Baumwolle ein), Kautschuk und Kupfer — vom Auslande unabhängig zu machen, ferner die Möglichkeit der A u $\mathrm{f} \mathrm{u} \mathrm{h} \mathrm{r}$ deutscher Industrieprodukte nach den Kolonien einheitlich zu behandeln ist Aufgabe des Technologen ${ }^{4}$ ). Es sollten daher in jeder technologischen Sammlung auch die Kolonien vertreten sein.

Auf dem verschlossenen Vorplatz sind in Schränken Bücher und Zeitschriften, Zeichnungen, Tabellen u. dgl. (ebenfalls vom Verf. geschenkt) aufgestellt.

Eine solehe chemisch-technologische Sammlung laßt sich allerdings nicht vom $\mathrm{S} \mathrm{ch} r$ e i b t is ch aus durch einige Briefe an Fabriken zusammenbringen, sondern nur durch langjährigen Verkehr mit Technikern und häufige längere Besuche von Fabriken. Sie muß ferner fortwährend ergänzt und erweitert werden, um ein möglichst getreues Bild von dem tatsächlichen Stande der Industrie zu geben, was nuŕ ein erfahrener Fachmann kann. - Auf die große Bedeutung einer solchen Sammlung $^{5}$ ) für Chemiker, Juristen ${ }^{6}$ ) und Lehramtskandidaten muß verwiesen werden.

Für die Beschaffung der erforderlichen Schränke wurden von der ,Göttinger Vereinigung“" $600 \mathrm{M}$, von Hannoverschen Fabriken $330 \mathrm{M}$ geschenkt; das übrige habe ich geliefert, auch die ganze Arbeit.

Der Wert der Sammlung beträgt etwa $10000 \mathrm{M}$.

\section{Beitrag zur Bestimmung der Alkalien im Trinkwasser.}

Mitteilung aus dem staatl. hygienischen Institut zu Hamburg. - Direktor Prof. Dr, Dunbar.

\section{Von Erich Koch.}

(Eingeg. d. 8./6. 1909)

Die geringen Mengen Alkalien, die ein gutes Trinkwasser gelöst enthält, sind in hygienischer Beziehung von keinem Interesse, es sei denn, daß sie an Kohlensäure gebunden das wirksame Prinzip der Säuerlinge darstellen. Deshalb sieht man meist von einer Einzelbestimmung der Alkalien ab. Wird dagegen der Gehalt an Natrium- und Kaliumchlorid durch besondere Bodenformation, durch Abfallstoffe des mensehlichen Haushalts oder durch Fabrikabwässer erheblich erhöht, so sieht man sich vielfach veranlaßt, eine quantitative Trennung der Alkalien vorzunehmen.

Diese Bestimmungen sind nach den bekannten Methoden teils direkt, teils indirekt, mittels $\mathrm{Be}$ rechnung ausführbar.

Die eine besteht darin, daß man die schwefelsauren Alkalien bestimmt und hierin die Schwefelsäure. Aus letzterer wird die vorhandene Menge

4) Vgl. Ferd. Fischer, Die Industrie Deutschlands und seiner Kolonien. 2. Aufl. (Leipzig 1908).

5) F is c h e r, Technologie für Chemiker und Juristen an den preußischen Universitäten. (Leipzig 1903). S. 15.

6) Diese Z. 1908, $196 \%$.
Kaliumsulfat und Natriumsulfat berechnet1). Dieses Verfahren liefert aber nach den Versuchen von

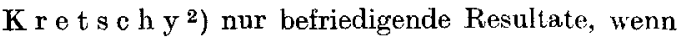
$\mathrm{Na}_{2} \mathrm{SO}_{4}$ in vorwiegender Menge vorliegt. Sind gleiche Teile vorhanden, oder wiegt $\mathrm{K}_{2} \mathrm{SO}_{4}$ vor, so sind die Ergebnisse ungenau und nicht brauchbar.

Anderer Art ist die Methode von E. $\mathrm{BOh} \mathrm{li} \mathrm{g}^{3}$ ):

Man verdampft $500 \mathrm{com}$ Wasser auf $50 \mathrm{ccm}$, versetzt es mit konz. $\mathrm{H}_{2} \mathrm{SO}_{4}$ bis zur starksauren Reaktion und dampft es bis zum Entweichen von Schwefelsäuredämpfen ein. Dann spült man mit $150 \mathrm{ccm}$ Wasser in ein Kölbchen, gibt aufgeschwemmtes kohlensaures Barium zu und leitet $\mathrm{CO}_{2}$ bis zur Sättigung ein. Man filtriert, wäscht gut aus, dampft bei $130-140^{\circ}$ ein und digeriert den Rückstand mit $50 \mathrm{com}$ einer Mischung gleicher Teile Alkohol und Wasser, wodurch nur die Alkalicarbonate aufgenommen werden. Nach dem Filtrieren titriert man mit 1/10-n. Säure und erfährt so den Gesamtgehalt an Chloralkalien. Die Trennung dieser findet nach der später angeführten Platinchloridmethode statt.

Nach einer indirekten Trennungsvorsohrift $\left.{ }^{\star}\right)$, deren Resultate nach $\mathrm{L}$ u n g e auf große Genauigkeit keinen Anspruch erheben, wird im Wasser Kalk und Magnesia, festgebundene Kohlensäure, Salpetersäure und Chlor bestimmt und dann die Menge der Alkalien berechnet, indem man die gefundenen Mengen der Basen und die der Säuren durch ihr Äquivalentgewicht dividiert, dann die Quotienten der Basen und die der Säuren für sich addiert. Enthält das Wasser Alkalien, so ist die Summe der Quotienten der Basen kleiner, als die derjenigen der Säuren Multipliziert man die Differenz mit dem Äquivalentgewicht des Natrons, so erhält man die Menge der Alkalien in Natron ausgedrückt.

Die folgende Bestimmung ${ }^{5}$ ) ist die bekannteste, sie liefert sehr gute Resultate. Man fällt in der Wasserprobe die $\mathrm{H}_{2} \mathrm{SO}_{4}$ mit $\mathrm{BaCl}_{2}$, versetzt das Filtrat mit Bariumhydrat bis zur stark alkalischen Reaktion. Nachdem hierdurch Fisen, Aluminium und Magnesium ausgeschieden sind, fällt man Kalk, Baryt und einen Rest von Magnesia mit Ammoniumcarbonatlösung. Das Filtrat dampft man wiederholt mit einigen Tropfen Ammoniumoxalat ein, bis aller Kalk und Baryt entfernt ist, vertreibt durch mäßiges Erhitzen die Ammoninmsalze, nimmt wieder mit Wasser auf, filtriert, dampft unter $\mathrm{Zu}$. satz von wenig Salzsäure zur Trockne, glüht bei schwacher Rotglut und wägt die Gesamtalkalichlormetalle. Zur Trennung der Alkalichloride löst man sie in wenig Wasser, setzt Platinchlorid zu und verdampft in einer Porzellanschale bis zur Sirupdicke, gibt $80 \%$ igen Alkohol zu, $\mathrm{Na}_{2} \mathrm{PtCl}_{6}$ geht in Lösung, $\mathrm{K}_{2} \mathrm{PtCl}_{6}$ scheidet sich ab und wird am besten in einem $\mathrm{Gooch} \mathrm{schen} \mathrm{Tiegel} \mathrm{mit} \mathrm{Asbest}$ abgesaugt. Man troeknet nach dem Auswaschen mit Alkohol und Äther bis zur Gewichtskonstanz,

i) Classen, Handb. d. anizl. Chem. 5, 107

2) Kretschy, Z. f. anal. Chem. I5, 56.

3) E. Bohlig, Z. f. anal. Chem. 38, 431 bis 432 .

4) Lunge, Chem. techn. Untersuchungsmethoden 1, 800 [1904].

5) Vgl. auch Tiemann-Gärtner, 4. Aufl. 1895 , S. 108 . 
wägt, löst mit heißem Wasser $\mathrm{K}_{2} \mathrm{PtCl}_{6}$ heraus, trocknet, wägt wieder; die Differenz ergibt die Menge des $K_{2} \mathrm{PtCl}_{6}$, welches auf $\mathrm{KCl}$ umgereehnet wird. $\mathrm{KCl}$ von den Gesamtalkalichloriden subtrahiert, ergibt den Gehalt an NaCl.

Diese Trennung ist etwas umständlich und zeitraubend, man hat deshalb auf kürzerem Wege die einzelnen Alkalichloride durch Berechnung zu ermitteln versucht $\left.{ }^{6}\right)$. Man verfährt bis zur Bestimmung der Gesamtalkalichloride in der oben geschilderten Weise, danach stellt man den Gehalt des Chlors titrimetrisch oder auch gewichtsanalytisch mit $\mathrm{AgNO}_{3}$ fest. Bezeichnet man mit $\mathrm{S}$ die Summe von $\mathrm{KCl}$ und $\mathrm{NaCl}$, mit $\mathrm{O}$ die gefundene Menge von Chlor, so ist:

$$
\begin{gathered}
\mathrm{x}(\mathrm{KCl})=4,6413(\mathrm{~S}-1,6502 \mathrm{C}) \\
\mathrm{y}(\mathrm{NaCl})=\mathrm{S}-\mathrm{x} .
\end{gathered}
$$

Diese Zahlen erklären sich aus folgendem ${ }^{7}$ ):

Nennt man die Summe der Chlormetalle = a, die Menge des Chlors $=b$, so ist, wenn man die Menge des Chlors ganz auf Chlorkalium berechnet, entsprechend den Molekulargewichten

$$
35.5: 74,6=b: x \text {. }
$$

Man erhält so die Zahl c, die größer ist, als das Gewicht der gewogenen Gesamtalkalichlormetalle, da das Molekulargewicht des $\mathrm{Na}$ kleiner ist als das des K. Der Unterschied c - a steht zur Menge des $\mathrm{Na}$ in demselben Verhältnis, in dem 16, die Differenz zwischen den Molekulargewichten der Chlormetalle (74,5-58,5), zu dem Molekulargewicht des Chlornatriums steht. Nach der Proportion

$$
16,1: 58,5=c-a: x,
$$

ist $\mathrm{x}$ die Menge des $\mathrm{NaCl}$ in der Verbindung.

Diese indirekte Methode ist zwar theoretisch richtig, sie liefert jedoch in praxi in den seltensten Fällen richtige Werte.

Angenommen man hat $400 \mathrm{mg}$ Gesamtalkalien, bestehend aus $200 \mathrm{mg} \mathrm{KCl}$ und $200 \mathrm{mg} \mathrm{NaCl}$, und man hätte genau die entsprechenden $216,4 \mathrm{mg} \mathrm{Cl}$ gefunden, so würde man nach obiger Berechnung richtig $200 \mathrm{mg} \mathrm{NaCl}$ finden und nach Subtraktion von $400 \mathrm{mg}$ Gesamtalkalichloride $200 \mathrm{mg} \mathrm{KCl}$.

Nun ist zu bedenken, daß man oft trotz mehrmaligen Eindampfens mit Ammoniumoxalat Spuren von $\mathrm{BaCl}_{2}$ mit zur Wägung der Gesamtalkalien bringt oder andere kleine Differenzen erhält, dio noch ganz in der Fehlergrenze liegen, z. B. statt $400 \mathrm{mg} \mathbf{4 0 2} \mathrm{mg}$ wägt, in diesem Falle erhält man als Resultat $192,6 \mathrm{mg}$ für $\mathrm{NaCl}$, statt $200 \mathrm{mg}$ und $209,4 \mathrm{mg} \mathrm{KCl}$ statt $200 \mathrm{mg}$.

Oder aber: Die Befun?le der Gesamtchloralkalien seien richtig $400 \mathrm{mg}$, jedoch das gefundene Chlor z. B. $218,4 \mathrm{mg}$ statt 216,4 , so ist das Ergebnis dieser indirekten Bestimmung $215,3 \mathrm{mg}$, statt $200 \mathrm{mg}$ $\mathrm{NaCl}$ und 184,7 $\mathrm{mg}$ statt $200 \mathrm{mg} \mathrm{KCl}$. Derartige falsche Resultate veranlaßten mich, über die Brauchbarkeit dieser rechnerischen Trennung Versuche anzustellen.

Im folgenden soll nun an der Hand mehrerer Tabellen gezeigt werden, inwieweit die Resultate der indirekten Methode von denen der exakten Platinchloridtrennungsart verschieden sind. Ich benutzte zu diesen Analysen zunächst nur verschiedene Lösungen bekannten Gehalts von $\mathrm{KCl}$ und $\mathrm{NaCl}$. Trotzdem wurde der oben angegebene Analysengang innegehalten, d. h. die salzsaure Lösung mit Bariumhydrat, Ammoniumcarbonat usw. behandelt. Das Chlor wurde sowohl gewichtsanalytisch wie

\begin{tabular}{|c|c|c|c|}
\hline & $\begin{array}{l}\text { Gesamt- } \\
\text { Alkaliehloride }\end{array}$ & Kaliumehlorid & Natriumehlorid \\
\hline Die künstliche Lösung enthielt in $500 \mathrm{ccm}: \cdot .\{$ & $\begin{array}{l}\text { I. } 400,6 \mathrm{mg} \\
\text { II. } 400,6,\end{array}$ & $\begin{array}{l}\text { I. } 199,6 \mathrm{mg} \\
\text { II. } 199,6 \text { r }\end{array}$ & $\begin{array}{l}\text { I. } 201,0 \mathrm{mg} \\
\text { II. } 201,0 \text {, }\end{array}$ \\
\hline Gefunden ( $\mathrm{KGl}$ aus $\mathrm{K}_{2} \mathrm{PtCl}_{6}$ berechnet): . & $\begin{array}{l}\text { I. } 403,5 \mathrm{mg} \\
\text { II. } 405,5,\end{array}$ & $\begin{array}{l}\text { I. } 201,4 \mathrm{mg} \\
\text { II. } 201,5,\end{array}$ & $\begin{array}{l}\text { I. } 202,1 \mathrm{mg} \\
\text { II. } 203,9 \quad,\end{array}$ \\
\hline Gefunden (indirekte Methode): . . . . . . , \{ & $\begin{array}{l}\text { I. } 403,5 \mathrm{mg} \\
\text { II. } 405,5,\end{array}$ & $\begin{array}{l}\text { I. } 271,0 \mathrm{mg} \\
\text { II. } 261,9 \text {, }\end{array}$ & $\begin{array}{l}\text { I. } 132,4 \mathrm{mg} \\
\text { II. } 143,6 \quad\end{array}$ \\
\hline
\end{tabular}
titrimetrisch ermittelt. Die angewendeten Chemikalien waren chemisch rein.

Tabelle I.

Die gewogenen Gesamtchloralkalien wurden gelöst zu $100 \mathrm{ccm}$ und in je $50 \mathrm{ccm}$ einmal die Trennung mit $\mathrm{H}_{2} \mathrm{PtCl}_{6}$, das andere $\mathrm{Mal}$ mittels Berechnung aus dem gefundenen Chlor bewerkstelligt. Wie aus der Tabelle zu ersehen ist, wurde bei der indirekten Methode $71,4 \mathrm{mg}$, bezüglich $62.3 \mathrm{mg} \mathrm{KCl}$ zuviel und 68,6 bzw. $57,4 \mathrm{mg} \mathrm{NaCl}$ zuwenig gefunden. Durch Multiplikation der kleinen Fehler mit einem hohen Faktor werden diese erheblichen Differenzen verursacht.

6) Classen, 5. Aufl. 1900, Handb. d. quant. Analyse, S. 105.

7) N. Menschutkin, Anal. Chem. 3. Aufl. 1892, S. 300; vgl. auch Muspratts Chemie, 5. Aufl. 1893, Bd. 4, S. 829 .
Bei diesen drei Analysen fällt zunächst der etwas hohe Befund der Gesamtalkalien auf, der wohl durch Verunreinigung von Spuren des hochmolekularen $\mathrm{BaCl}_{2}$ zu erklären ist oder aber durch die Art des Glühens der Chloride. Einige Analytiker schreiben ein Erhitzen bis zur sohwachen Rotglut vor, andere bis zum beginnenden Schmelzen des: Salzes, letztere Handhabung liefert meist bessere Resultate.

Bei diesen Analysen enthielt die Lösung 3,3mal soviel $\mathrm{NaCl}$ als $\mathrm{KCl}$, die Resultate der indirekten Methode sind falsch.

Diese drei Lösungen enthielten die 3,3fache Menge $\mathrm{KCl}$ im Verhältnis zu NaCl. Die Resultate sind ebenfalls unbrauchbar, wenngleich sie besser sind als diejenigen der Tabelle II. 
T'abelle II.

\begin{tabular}{|c|c|c|c|c|}
\hline & & $\begin{array}{l}\text { Geaamt- } \\
\text { Alkalichloride }\end{array}$ & Kaliumchlorid & Natriumchlorid \\
\hline Die künstliche Lösung enthielt in $500 \mathrm{ccm}$ : & $\cdot \cdot\{$ & $\left.\begin{array}{l}\text { I. } \\
\text { II. } \\
\text { III. }\end{array}\right\} 130,0 \mathrm{mg}$ & $\left.\begin{array}{r}\text { I. } \\
\text { II. } \\
\text { III. }\end{array}\right\} 30 \mathrm{mg}$ & $\left.\begin{array}{r}\text { I. } \\
\text { II. } \\
\text { III. }\end{array}\right\} 100 \mathrm{mg}$ \\
\hline Gefunden ( $\mathrm{KCl}$ aus $\mathrm{K}_{2} \mathrm{PtCl}_{B}$ berechnet): . & . & $\begin{aligned} \text { I. } & 135,0 \mathrm{mg} \\
\text { II. } & 133,0 \quad " \\
\text { III. } & 134,0 \quad "\end{aligned}$ & $\begin{aligned} \text { I. } & 28,99 \mathrm{mg} \\
\text { II. } & 26,2 \quad " \\
\text { III. } & 27,5 \quad "\end{aligned}$ & $\begin{array}{rr}\text { I. } & 106,0 \mathrm{mg} \\
\text { II. } & 106,8 \% \\
\text { III. } & 106,5\end{array}$ \\
\hline Gefunden (Indirekte Methode): & . . & $\begin{aligned} \text { I. } & 135,0 \mathrm{mg} \\
\text { II. } & 133,0 " \\
\text { III. } & 134,0,\end{aligned}$ & $\begin{array}{rll}\text { I. } & 78,81 \mathrm{mg} \\
\text { II. } & 55,2 \\
\text { III. } & 57,3\end{array}$ & $\begin{array}{rlr}\text { I. } & 56,19 \mathrm{mg} \\
\text { II. } & 77,8 \quad " \\
\text { III. } & 76,7\end{array}$ \\
\hline
\end{tabular}

Tabelle III.

\begin{tabular}{|c|c|c|c|}
\hline & $\begin{array}{l}\text { Gesamt- } \\
\text { Alkalichloride }\end{array}$ & Kaliumchlorid & Natriumchlorid \\
\hline Die künstliche Lösung enthielt in $500 \mathrm{ccm}$ : & $\left.\begin{array}{l}\text { I. } \\
\text { II. } \\
\text { III. }\end{array}\right\} 130 \mathrm{mg}$ & $\left.\begin{array}{r}\text { I. } \\
\text { II. } \\
\text { III. }\end{array}\right\} 100 \mathrm{mg}$ & $\left.\begin{array}{l}\text { I. } \\
\text { II. } \\
\text { III. }\end{array}\right\} 30 \mathrm{mg}$ \\
\hline Gefunden ( $\mathrm{KCl}$ aus $\mathrm{K}_{2} \mathrm{PtCl}_{6}$ berechnet): . . & $\begin{aligned} \text { I. } & 134,8 \mathrm{mg} \\
\text { II. } & 134,4 \mathrm{n} \\
\text { III. } & 134,6 \quad\end{aligned}$ & $\begin{array}{rcc}\text { I. } & 100,6 \mathrm{mg} \\
\text { II. } & 99,13 " \\
\text { III. } & 99,8\end{array}$ & $\begin{aligned} \text { I. } & 34,2 \mathrm{mg} \\
\text { I.I. } & 35,2 \quad " \\
\text { III. } & 34,8 \quad "\end{aligned}$ \\
\hline Gefunden (Indirekte Methode): & $\begin{array}{rr}\text { I. } & 134,8 \mathrm{mg} \\
\text { II. } & 134,4 \quad " \\
\text { III. } & 134,6 \quad "\end{array}$ & $\begin{aligned} \text { I. } & 126,8 \mathrm{mg} \\
\text { II. } & 116,5 \Rightarrow \\
\text { III. } & 120,4 \Rightarrow\end{aligned}$ & $\begin{array}{r}\text { I. } \quad 8,0 \mathrm{mg} \\
\text { II. } \quad 17,9 " \\
\text { III. } \quad 14,2 \%\end{array}$ \\
\hline
\end{tabular}

Tabelle IV zeigt die Ergebnisse der Alkalichloridanalyse in einem künstlichen Wasser, das aus $\mathrm{KCl}, \mathrm{NaCl}, \mathrm{MgCl}_{2}, \mathrm{Mg}\left(\mathrm{HCO}_{3}\right)_{2}, \mathrm{CaSO}_{4}, \mathrm{Ca}\left(\mathrm{HCO}_{3}\right)_{2}$ hergestellt wurde. $1000 \mathrm{ccm}$ enthielten:

$170 \mathrm{mg}$ Alkalichloride ( $50 \mathrm{mg} \mathrm{KCl}+120 \mathrm{mg} \mathrm{NaCl}$ ),

$58,5, \mathrm{CaO}$,

57,3 , $\mathrm{MgO}$,

$88,0, \mathrm{SO}_{3}$,

133,9 , Cl.

Zuerst wurde nach dem Ansäuern mit Salzsäure die Schwefelsäure mit $\mathrm{BaCl}_{2}$ ausgefällt, filtriert und mit Bariumhydrat, Ammoniumcarbonat usw. in bekannter Weise weiterbehandelt. Die Gegenwart anderer Salze beeinflubte die Isolierung der Chloralkalien nicht, wie die guten Resultate der Platinchloridmethode beweisen, die indirekte Methode verursachte Differenzen von 5,9-21,8.

Bei solchen durchaus fehlerhaften Ergebnissen der rechnerischen Trennung von Kalium- und $\mathrm{Na}$ triumchlorid kann diese für die quantitative Analyse nicht benutzt werden, am geeignetsten erscheint dafür die chemische Bestimmung mit Platinchlorid.

Tabelle IV.

\begin{tabular}{|c|c|c|c|}
\hline & $\begin{array}{c}\text { Gesamt. } \\
\text { Alkalichloride }\end{array}$ & Kaliumchlorid & Natriumchlorid \\
\hline Die künstliche Lösung enthielt im Liter: & $\left.\begin{array}{r}\text { I. } \\
\text { II. } \\
\text { III. }\end{array}\right\} 170 \mathrm{mg}$ & $\left.\begin{array}{r}\text { I. } \\
\text { II. } \\
\text { III. }\end{array}\right\} 50 \mathrm{mg}$ & $\left.\begin{array}{r}\text { I. } \\
\text { II. } \\
\text { III. }\end{array}\right\} 120 \mathrm{mg}$ \\
\hline Gefunden ( $\mathrm{KCl}$ aus $\mathrm{K}_{\mathbf{2}} \mathrm{PtCl}_{6}$ berechnet): . . & $\begin{array}{rrr}\text { I. } & 173,4 \mathrm{mg} \\
\text { II. } & 171,1 \% \\
\text { III. } & 172,3 & \end{array}$ & $\begin{aligned} \text { I. } & 50,5 \mathrm{mg} \\
\text { II. } & 51,3 \% " \\
\text { III. } & 53,0 \%\end{aligned}$ & $\begin{array}{rr}\text { I. } & 122,9 \mathrm{mg} \\
\text { II. } & 119,8 \\
\text { III. } & 119,3\end{array}$ \\
\hline Gefunden (Indirekte Methode): . . . . . . . . & $\begin{aligned} \text { I. } & 173,4 \mathrm{mg} \\
\text { II. } & 171,1 \text { " } \\
\text { III. } & 172,3 \text {, }\end{aligned}$ & $\begin{aligned} \text { I. } & 44,1 \mathrm{mg} \\
\text { II. } & 36,3 " \\
\text { III. } & 30,5 \%\end{aligned}$ & $\begin{array}{rr}\text { I. } & 129,3 \mathrm{mg} \\
\text { II. } & 134,8 \text { " } \\
\text { III. } & 141,8\end{array}$ \\
\hline
\end{tabular}

